\title{
Açaí, Euterpe oleracea: Rethinking Treatment for Inflammatory Conditions
}

Amanda Relph and Todd Pesek*

Department of Health Sciences and Center for Healing Across Cultures, Cleveland State University, Cleveland, Ohio, USA

\begin{abstract}
Inflammatory conditions affect individuals of all ages, races, and genders and originate from a relatively unknown combination of biological and environmental factors. Despite their prevalence, research has fallen short in providing significant discoveries in regards to both their etiology and effective treatment. However, there may be a relatively simple, efficacious treatment strategy involving the drupe of the acaí palm, Euterpe oleracea, more commonly known as acaí. Overwhelming evidence suggests that consumption of the acaí fruit in the appropriate form and concentration represents an avenue of or adjunct to treatment for inflammatory conditions not previously applied. This particular systematic review evaluated the potential efficacy of utilizing E. oleracea in the treatment of three representative and common inflammatory conditions with similar cellular pathophysiology -- Fibromyalgia Syndrome, Chronic Fatigue Syndrome, and Rheumatoid Arthritis.
\end{abstract}

Keywords: Acaí; Anthocyanins; Antioxidant Activity; Chronic Fatigue Syndrome; Cytoprotection; Euterpe Oleracea; Fibromyalgia; Immunomodulation; Inflammatory Response; Rheumatoid Arthritis

\section{Introduction}

Each year, physicians across the country experience an influx of patients with complaints of chronic pain. These patients often present with symptoms including, diffuse myalgias and/or arthralgias, fatigue, stiffness, sleep disturbances, and high incidence of comorbidities $[1,2]$. Often, these symptoms progress for months to years without successful diagnostic investigation, proper diagnosis, or relief via treatment. A simple solution may be emerging since research shows that there may be an efficacious treatment strategy involving the fruit of the acaí palm, Euterpe oleracea, commonly known as acaí. Based on these newly emerging data, a systematic review of the literature to evaluate the efficacy of utilizing E. oleracea in the treatment of select inflammatory conditions, including Fibromyalgia Syndrome, Chronic Fatigue Syndrome, and Rheumatoid Arthritis was performed. These particular conditions were chosen because they are representative of prevailing diagnoses in inflammatory conditions. We present the review data following.

\section{Methods}

\section{Study design}

A systematic review was used to conduct the research. In doing so, historical data and scientific information specific to substance properties and bioactivity were collected and reviewed for reliability, validity, and authenticity; this data was then grouped according to key points, and a conceptual hypothesis was formed. Historical research was also conducted in the examination of various inflammatory conditions; information gathered includes signs, symptoms, laboratory tests and values, involvement of multiple organ systems, and other pertinent information.

\section{Data collection}

Data and theoretical information regarding E. oleracea and its properties were obtained through comprehensive literature review. In addition, review and analysis of the integrity of numerical and statistical data was performed with regard to the substance's biological and chemical properties and its effects on multiple organ systems (i.e. polyphenolic concentration, free-radical scavenging activity, cell mortality). Defining characteristics (i.e. laboratory values and biochemical considerations), pathogenicity, and typical clinical presentation of several inflammatory conditions were also explored.
Information was obtained primarily from peer-reviewed journals and scholarly medical publications, including published case studies; a small amount of information was also gathered from commercial presentations relaying basic knowledge of the substance, such as origin and historical significance.

In systematic literature review, three levels were employed: initial, level one, and level two. In the initial review stage, all potential sources of information were evaluated for applicability to the research subject; those that focused on information irrelevant or inapplicable were excluded. Sources found to meet the initial criteria for review underwent a level one review, in which all information was evaluated for continuity across sources; the primary goal at this level was to identify inconsistencies in research and results. Finally, all remaining sources underwent the second level of systematic review - an evaluation of quality. It was at this level that qualitative/quantitative data soundness and the rigor with which they were collected and analyzed was examined. Sources that failed to utilize adequate sample sizes, randomization techniques, and bias-controlling measures were excluded, and those that remained were ranked in terms of relevance and quality. Based upon the remaining information, a hypothesis regarding the biological activity of acaí extract and its potential effectiveness in the treatment of select inflammatory conditions was made for theoretical consideration.

\section{Trustworthiness \& authenticity}

Throughout the literature review, characteristics that remained the same trans-study were documented and examined in order to ensure data integrity and reliability. Scientific concepts and statistical data were included only from peer-reviewed, published, credible sources. The researcher considered scientific rigor when examining the processes with which the published researchers conducted their studies; likewise, rigor was employed during analysis of the resultant data and findings.

*Corresponding author: Todd Pesek, Department of Health Sciences and Center for Healing Across Cultures, Cleveland State University, 2121 Euclid AvenueHS101, Cleveland, Ohio 44115, Tel. 216-523-7353, Email: t.pesek@csuohio.edu

Received October 20, 2011; Accepted December 06, 2011; Published December 09, 2011

Citation: Relph A, Pesek T (2011) Açaí, Euterpe oleracea: Rethinking Treatment for Inflammatory Conditions. J Nutr Disorders Ther 1:103. doi:10.4172/2161 0509.1000103

Copyright: $\odot 2011$ Relph A, et al. This is an open-access article distributed under the terms of the Creative Commons Attribution License, which permits unrestricted use, distribution, and reproduction in any medium, provided the original author and source are credited. 
Triangulation of one study against another was used often to examine the conceptual basis of the research question, and negative case analysis was performed on several occasions in order to compare and contrast various findings.

\section{Fibromyalgia Syndrome}

Fibromyalgia syndrome (FMS), perhaps one of the most commonly-diagnosed chronic musculoskeletal disorders, represents a group of signs and symptoms typically ranging in severity that include fatigue; chronic, widespread pain; prevalence of localized tender points; mild to moderate impairment of cognitive processes; disruption in sleep patterns; and, often, depression and/or anxiety [4-7]. According to Murphree, "Over 10 million Americans suffer from fibromyalgia; $90 \%$ of them are women between the age of 25 and 40 " [4]. Researchers conclude that women are significantly more likely than men to develop FMS, though the reason for this difference is largely unknown. Contrary to popular belief, the development of the disease in both sexes has been only minimally correlated with stressful life situations [6]. Historically, FMS has commonly been misconstrued as a "catch-all" diagnosis for unexplained musculoskeletal symptoms. Science has proven, however, that FMS elicits very specific abnormal laboratory findings in several areas, including cellular characteristics, immunological activity, and certain components of the central nervous system [3, 5-16].

\section{Etiology}

To date, the cause of FMS is largely unknown; however, several key factors have been identified in its presentation and progression $[11,12]$. For years, scientists suggested that severely traumatic events, both physical and emotional, played a significant role in the existence of FMS; this phenomenon was most commonly associated with war veterans and was subsequently termed Gulf War Syndrome [17]. Recently, however, researchers have begun to unearth perhaps some of the most significant etiological information regarding FMS thus far.

Sleep abnormalities and hormone dysregulation: According to researchers at the University of Maryland Medical Center, patients suffering from FMS commonly experience chronic sleep disturbances, which amplify existing symptoms, including pain and fatigue: “... patients with fibromyalgia have increased rates of cyclic alternating sleep pattern (CAP). Increased CAP produced serious sleep problems, which were strongly linked to symptom severity" [5]. Often linked to the disturbance or absence of deep sleep, hormonal dysregulation seems to play a part in FMS progression; in fact, recent studies have shown that FMS patients tend to also suffer from inadequate amounts of serotonin, cortisol, norepinephrine, dopamine, growth hormone $(\mathrm{GH})$, and insulin-like growth factor (IGF-1) $[3,5,7,13,15]$. Many of these deficiencies are thought to contribute to the hallmark symptoms of the disease; consider, for example, the fact that serotonin works closely with norepinephrine in the CNS to control the processing of pain signals [7]. Decreased serotonin levels, therefore, would alter the central nervous system's ability to process pain effectively. Additionally, the precise balance of serotonin and dopamine has proven vital for healthy sleep patterns, and cortisol and norepinephrine work closely to control the adrenals; thus, abnormalities in these areas would alter the body's ability to sustain energy and combat fatigue. Perhaps even more important is the relationship between low GH, low IGF-1, and the immune system's incapacity to repair damaged tissue and/or dispose of dead cellular matter [18]. Lastly, and perhaps most commonly noted by researchers and medical practitioners alike, a large percentage of FMS patients also suffer from a significant lack of Vitamin D. Studies indicate that this deficiency affects the body's ability to regulate calcium concentrations, particularly in the bones and muscles, contributing significantly to muscle contractility and diffuse pain, dysregulation of the immune system, and inability to combat chronic inflammation [1922] - all of which are hallmark characteristics of FMS.

CNS abnormalities: CNS abnormalities have been identified in a large percentage of FMS patients. Perhaps the most significant finding in this realm involves the discovery of elevated CSF concentrations of nerve growth factor (NGF) and substance P $[3,5-7,16,23]$. Both substances significantly increase the body's sensitivity to pain, and many studies have found the concentrations of these chemicals in FMS patients to be up to four times higher than those of healthy individuals [3-5,7].

Research shows that CNS abnormalities in FMS hinge also on the chronic dysfunction of the hypothalamic-pituitary-adrenal (HPA) axis. The HPA axis serves primarily to maintain homeostasis within the body and does so largely via the stimulation, production, and circulation of hormones [3, 4]. Science has deemed the HPA axis one of the most influential factors in the existence and progression of FMS; in fact, theories suggest that its dysregulation may be one of the primary causative agents for the disease, resulting in many of the hormone abnormalities common in its progression $[3,4,6,7]$.

Molecular and cellular abnormalities: In terms of cellular structure, research indicates that individuals with FMS possess very specific abnormalities at the molecular level - fragmented DNA, absence of cell apoptosis, and low levels of cellular mitochondria - that, in combination, contribute to the fatigue, myalgia, and inflammatoryderived symptoms characteristic of the condition $[9,10]$.

DNA fragmentation equates to damaged cells and related components, which, in healthy individuals, results in apoptosis. However, FMS patients seem to lack the ability for apoptosis; subsequently, this continual cellular damage stimulates the release of inflammatory cytokines IL-1Ra, IL-8, IL-6, and TNF- $\alpha$, initiating a wide-spread, uninterrupted inflammatory process that results in diffuse pain and increased nociception [8]. These particular cytokines also affect the hypothalamus [24]; coupled with decreased ATP production due to inadequate cellular mitochondria, the two tend to produce severe and prolonged fatigue.

Immunological Abnormalities: Researchers have long been divided on the subject of immunological involvement in FMS; some studies strongly suggest an immune component, while others negate it altogether. However, the discovery of elevated concentrations of inflammatory cytokines indicate that FMS patients do, in fact, possess some sort of immunological dysfunction [8, 14]. More recently, scientists have discovered the presence of anti-polymer antibodies (APAs) in FMS patients. These antibodies are thought to form in response to the disease itself. Though their mechanism of formation and physiological purpose are unknown to date, the APA assay has recently been proposed as the first concrete laboratory test for FMS [25], as these antibodies are not present in those suffering from similar conditions $[3,11,12]$.

Free Radicals: Perhaps one of the most consistent concepts in FMS research is that of ROS involvement. Science suggests that FMS may develop, partially, as a result of high levels of oxidative stress in the body [9]. In several studies, FMS patients have been found to possess extremely high levels of particular free radicals and significantly low levels of SOD, the body's most powerful antioxidant [9]. In addition, those with the condition have been found to lack normal levels of $\mathrm{CAT}$, indicating an insufficient antioxidant capacity to combat the large generation of ROS and the subsequent oxidative damage taking place [26]. Many researchers have postulated that this ROS damage 
leads to tissue hypoxia, tender points, and diffuse myalgia, providing strong evidence that oxidative stress due to cellular damage, DNA fragmentation, and inflammation plays a key role in pathophysiology of the disease [9, 27-29].

\section{Chronic Fatigue Syndrome}

For years, chronic fatigue syndrome (CFS) has remained somewhat of a mystery to conventional medical practitioners. The disease often presents as an array of symptoms that can often be mistaken for or attributed to many temporary, less severe ailments; in addition to the presence of "...unexplained fatigue lasting 6 months or more," a recurrent ebb and flow of genera, less-severe flu-like symptoms comprise the eight standard clinical indicators for suspected CFS [30, 31]. The disease affects millions annually, and research is ongoing in an attempt to discover a direct cause [32, 33].

\section{Etiology}

Sleep abnormalities and hormone dysregulation: The CFIDS Chronicle reports that, "Unrefreshing sleep is a major symptom of CFS, with 70-95\% of people with the illness reporting this symptom..." [34]. Though researchers have been unable to clarify whether or not sleep disturbance functions at the pathogenic level, science has confirmed a significant imbalance of the hormones related to healthy sleep patterns. For example, reports show that CFS patients lack adequate levels of dopamine, melatonin, cortisol, norepinephrine, and possess high levels of serotonin - all of which contribute to altered sleep patterns and feed directly into impaired CNS functionality $[31,34,35]$.

CNS Abnormalities: Research suggests that overstimulation of the sympathetic nervous system and dysfunction of the HPA axis operate as two of the most prevalent contributors to many of the hallmark signs and symptoms of the disease $[30,34,37]$. Chronic activation of the sympathetic nervous system puts the body in a continuous state of stress, requiring "... an exaggerated stress response" from the HPA axis [30]. This overstimulation of the HPA axis results in an eventual breakdown in its ability to assist in the maintenance of a homeostatic environment, and thus, plays a central role in the resulting in hormonal imbalance, fatigue, sleep disturbances, and the typical post-exertional malaise [30, 37].

Molecular and Cellular Abnormalities: At the forefront of CFS research is the existence of varying levels of mitochondrial damage, subsequent dysfunction, and disturbance in ATP production [34, 38]. Thought to arise from severe oxidative stress, mitochondrial membranes are compromised, thus decreasing the organelle's ability to produce and release ATP and NADH, two of the body's primary sources of energy. The damage typically extends beyond the membrane to the mitochondrial DNA, often permanently altering its capability to function at an optimal level (38). Such damage to the mtDNA severely impairs the body's capability for DNA repair; furthermore, mtDNA mutation has been linked to an array of serious diseases, such as a number of cancers, cyclic vomiting syndrome, and MELAS [39]. The extent of mitochondrial damage and DNA mutation, however, hits much closer to home for many, as science indicates that damageinduced ATP insufficiency is a significant contributor to CFS signs and symptoms: "Mental/emotional symptoms of poor attention, memory loss, lack of concentration, and depression may...be reflective of insufficient central nervous system ATP availability..." [40].

Immunological Abnormalities: A great deal of evidence exists implicating significant immunological involvement, primarily hyperactivity, in CFS patients [34, 41]. This results in the long-term, continual release of inflammatory cytokines and increased neutrophil apoptosis [34, 36, 38]. Laboratory tests confirm these findings, as initial tests for the condition often reveal elevated levels of CRP (c-reactive protein) and WBCs [41, 42]. Further testing often identifies an increase in various inflammatory cytokines, evidence of oxidative damage (via the presence of certain molecules, such as methemoglobin), and a marked decrease in antioxidant enzymes - a combination that produces sort of a domino effect, spreading throughout the body until fatigued and more susceptible to illness [38, 41]. In fact, medical science has recently unearthed several defining immunological components important to understanding CFS pathology, including decreased number of NK cells, increased proteolysis, and increased levels of NO [31]. Research continues to confirm that this chronic overstimulation of the immune system is directly related to the characteristics of the disease: "[The presence of] an immune disturbance... is in line with one favored theory that many of the symptoms of chronic fatigue syndrome derive from excessive cytokine release" [41].

Free Radicals: Similar to the findings in FMS research, studies show that oxidative stress via ROS production is key to understanding the pathogenesis of many of the symptoms common to CFS [43]. Kennedy et al. found that CFS patients carried particularly high levels of plasma-bound isoprostanes, chemical markers that indicate severe oxidative stress [36]. Additionally, their study suggested that those with the condition lack sufficient levels of antioxidants, making these individuals more susceptible to oxidative stress and damage. As researchers continue to discover the underlying pathology for the disease, many have deemed ROS a significant factor: "It could be suggested that CFS is an inflammatory condition with many patients in a prooxidant state, and this could explain many of the pathological manifestations that underlie the illness" [36].

\section{Rheumatoid Arthritis}

Scientifically proven to be a disease of autoimmune nature, RA has been termed a "chronic inflammatory polyarthritis," meaning that the disease produces ongoing (often fluctuating in severity) inflammation and pain in multiple joints throughout the body[44-46]. Research shows that RA tends to be more prevalent in the female population, and the risk for the development of the disease increases with age (peaking, then decreasing, near age 70). Recently, researchers have associated RA with a decrease in life expectancy and an increase in comorbidities and mortality dependent upon the severity of the disease [47].

\section{Etiology}

Though a concrete cause has not yet been identified, researchers have discovered several risk factors for the development of the disease, including an array of environmental and biological factors such as diet, tobacco use, hormonal influence, and genetics. Research also suggests that RA may have a viral component $[44,45,48,49]$. Science has recently uncovered a myriad of biological markers in patients suffering from the RA, providing us with key information and enhancing our understanding of the bioactivity and progression of the disease.

Sleep Abnormalities and Hormone Dysregulation: Much like its counterpart conditions, RA elicits significant signs and symptoms of sleep disturbance and hormone imbalance. In addition to chronic sleep abnormalities, patients suffering from the condition often lack normal levels of serotonin; this is especially important in understanding the intense pain experienced by many RA patients, as one of serotonin's many roles is the inhibition of substance $\mathrm{P}$ production $[50,51]$. Additionally, laboratory tests have confirmed that RA sufferers also experience significantly decreased levels of cortisol, stress hormones, and IGF-1 - substances that, at normal levels, serve to derail chronic 
inflammation and tissue damage [18, 51, 52]. Science has also pinpointed high dopamine levels and peaks and valleys in melatonin concentration as influential components in RA sleep disturbance [51, 53-55]. Perhaps one of the most significant discoveries in RA research, however, is that of consistently increased serum prolactin - a substance that, in high amounts, enhances chronic inflammatory response and increases one's risk for "the development of autoimmunity" [51, 56].

CNS Abnormalities: Research continues to implicate the involvement of abnormal CNS activity in RA existence and progression. Dekkers, Geene, Godaert, Bijlsma, and Van Doornen observed - an abnormal elevation in sympathetic nervous system (SNS) functionality with no increase in parasympathetic activity [57]. Additionally, Mukai et al. discovered a blatant HPA axis disturbance - now considered characteristic of the condition - evidenced not only by chronic sleep abnormalities, but also by consistently altered levels of key substances, such as serum prolactin, norepinephrine, dopamine, serotonin, and IGF-1 [50, 54, 56]. Increased levels and abnormal activity of substance $\mathrm{P}$ also play a significant role in RA existence and progression [58-60].

Molecular and Cellular Abnormalities: Science indicates that somatic mitochondrial mutation and DNA fragmentation and damage are characteristic of RA. Both Hitchon and El-Gabalawy and Da Sylva et al. discovered that both nuclear and mitochondrial DNA are severely damaged, resulting in the body's virtual inability to initiate and carry out DNA repair cycles, salvage its energy-building and releasing capabilities, protect itself from oxidative stress and further damage, and stave off chronic inflammation [45, 61]. In fact, researchers have indicated that this particular sort of damage to the mitochondria and DNA likely has the potential to produce very severe long-term effects: "If the mutations were in genes regulating cell survival, cells that would otherwise stop dividing and die (from DNA damage) may instead proliferate" [45].

Immunological Abnormalities: Research has long-since confirmed that, in those that suffer from RA, the immune system remains hyper-reactive and unable to recognize invader from self, attacking and eventually destroying healthy and functional tissue within the body [62-64]. Evidence also abounds for the involvement of inflammatory cytokines in the pathogenesis and progression of RA, particularly TNF- $a$, IL-1, and IL-6, often evidenced by high CRP levels [60, 64-68]. In addition, Da Sylva et al. and Cross et al. discovered a chronic pattern of abnormal cell apoptosis in RA patients, implicating and further proving the existence of chronic inflammation and tissue damage and altered immunoactivity $[45,68]$.

Free Radicals: ROS production occurs often in RA, producing significant and vast oxidative damage to important elements within the body, namely synovial tissue, lipids, proteins, and DNA[61]. In fact, research shows that those with the condition suffer high levels of lipid peroxidation and low levels of CAT and natural antioxidants - a recipe for chronic tissue damage, pain, and destruction of key functional structures $[45,59,61,70]$.

\section{The Acaí Fruit}

Native to South America, acaí, E. oleracea, is a palm tree belonging to the family Arecaceae [71]. The drupe of acaí, deep red or purple in color and composed of a large seed, thin layer of edible fruit, and a thick, fibrous skin, is harvested twice per year from tall, multi-stemmed palm branches [72]. Though little research has been conducted by way of human trials, scientists confirm that the fruit's pulp is rich in its applicability to medicine, health, and well-being. A staple of consumption in its indigenous environment, acaí is high in powerful antioxidants, valuable phytosterols, amino acids, and disease-fighting anthocyanins, and boasts an innate ability to combat inflammation, immunodeficiencies and abnormalities, chronic pain and disease, and, research suggests, even fight cancer [73-75]. It does so via: 1) antioxidant and enzyme-like activities, 2) immunomodulatory activity, 3) CNS regulation, 4) cellular repair, and 5) DNA repair mediation (Table 1).

\section{Antioxidant Properties and Enzyme-Like Activity}

FMS, CFS, and RA are similar in their ability and likelihood to produce high levels of ROS. Because ROS present a formidable threat to the healthy human body and potentiate the initiation and progression of chronic and debilitating disease, acaís ability to derail them via antioxidant content provides the possibility for substantial medical advancement in the way of non-pharmacological treatment. In fact, acaí boasts an Oxygen Radical Absorbance Capacity (ORAC) value of 1027, the highest value reported to date in comparison to other nutrient-dense fruits and vegetables. Researchers have discovered that the antioxidants present in acaí enter the cellular environment with ease, providing the body with intense free radical-fighting power [76].

Laboratory experiments confirm that the free radical-scavenging capability of E. oleracea far exceeds that of other comparable fruit extracts [71, 76-78]. Specifically, acaí prevents oxidative damage via its four distinct enzyme-like properties:

1. CAT - catalase, an enzyme that serves to protect cellular integrity via its ability to convert hydrogen peroxide to oxygen and water while avoiding the production of free radicals, is considered one of the most effective and efficient factors in the body's ability to detoxify its tissues [79]. CAT levels are extremely low in CFS, FMS, and RA alike, which likely serves as a partial explanation for the continual oxidative damage characteristic of the conditions. In laboratory experiments, studies continue to confirm that acaí possesses CATlike detoxification properties [77].

2. SOD - superoxide dismutase, an enzyme present in oxygenmetabolizing cells, acts to protect the body from free radical damage and has been shown to play a role in the reversal of fibrotic conditions [80; 81]. Research shows that patients with CFS, FMS, and RA are extremely SOD-deficient, also playing a key role in effects of ongoing ROS damage. Studies confirm that acaí possesses SOD-like capabilities, deeming it extremely effective in protecting the body from oxidative stress [77].

Table 1. Demonstrated benefits of açai (E. oleracea)

\begin{tabular}{ll}
\hline Antioxidant activity & $\begin{array}{l}\text { free radical scavenging } \\
\text { beneficial enzyme-like activity } \\
\text { inhibits excessive ROS production } \\
\text { antimicrobial characteristics } \\
\text { increased lymphocyte activity } \\
\text { prevention of immunosuppression } \\
\text { inhibits production of high levels of nitric oxide } \\
\text { anti-inflammatory activity } \\
\text { down-regulation of inflammatory cytokines } \\
\text { promotes healthy production of serotonin } \\
\text { promotes healthy production of dopamine } \\
\text { pain regulation } \\
\text { encourages homeostatic functionality of HPA axis } \\
\text { promotes appropriate substance P levels } \\
\text { encourages repair of damaged cell membranes } \\
\text { promotes optimal ATP production in mitochondria } \\
\text { promotes healthy function of DNA repair cycle }\end{array}$ \\
DNA repair mediation &
\end{tabular}

DNA repair mediation 
3. DPPH-scavenging - diphenylpicrylhydrazyl is a common free radical known to wreak oxidative havoc on the body and its tissues. Often, researchers measure an antioxidant's effectiveness based upon its ability to seek out and destroy the DPPH radical. Acaí ranks quite satisfactorily in comparison to others in its DPPHscavenging ability [77].

4. ROS inhibition - Reactive Oxygen Species are free radicals produced by the myriad of oxygen-metabolizing processes in the human body. While ROS can prove useful in certain immune processes in low concentrations, they can be extremely damaging to the body in large amounts; in fact, high concentrations of ROS can damage cell membranes, organelle structures, nucleic acids, and DNA strands [82]. In high doses, acaí has been shown to inhibit the production of large amounts of ROS, thus providing the body with molecular and cellular protection [71].

\section{Immunomodulatory Activity}

Given the evidence for abnormal immune activity in CFS, FMS, and RA, logical speculation suggests acaís promise in being utilized in treating them. Research confirms the immunosupportive properties of acaí , revealing not only its antimicrobial characteristics, but also its ability to protect the body's immune system via its ability to reduce oxidative stress [71, 78, 83]. Beta-sitosterol, one of acai's many components, has specifically been shown to prevent immune suppression and enhance lymphocyte activity [84]. Additionally, research confirms acaí's ability to inhibit unhealthy levels of nitric oxide production, a compound that plays a key role in immunosuppression when present in excessive concentrations $[85,86]$.

Anthocyanins, another major component of acaí , represent a strong group of antioxidants that also boast anti-inflammatory and analgesic properties [78, 87]. Cyanidin-3 glucoside, acaí's primary anthocyanin, has been shown to regulate pain and inflammation quite effectively. Many studies indicate the anthocyanins in acai to be highly effective, "...capable of attenuating inflammation... [and down-regulating] expression of enzymes involved in inflammation..." [78]. Other researchers have likened the activity of these substances to prescription-strength treatments for painful, inflammatory conditions: "... anthocyanins offer powerful relief against inflammation and pain... [and have] provided effective reduction of painful inflammation comparable to that obtained with the non-steroidal anti-inflammatory drug, Indomethacin..." [88]. Studies have also shown acaí to be effective in regulating the production of inflammatory cytokines, such as IL-Ra, IL-6, IL-8, and TNF- $\alpha$ common to all three conditions, thus controlling excessive inflammation to due cellular injury and/or damage $[77,78]$.

\section{CNS Regulation}

Research often speaks to acaí's ability to improve CNS operation, a component altered in those suffering from CFS, FMS, and RA. Acai, given its high concentration of B vitamins, has a proven capability to aid in the healthy production of serotonin and dopamine, two of the most vital neurotransmitters to CNS functionality, pain relief, and homeostasis [89]. Not only does this regulation stabilize mental and emotional functionality, it also provides for better sleep and total-body homeostasis via its encouragement of healthy HPA axis function and substance $P$ regulation $[50,51,53,90]$.

\section{Cellular Repair}

Predominant research suggests that a great deal of chronic disease is directly linked to cellular damage - often at the mitochondrial level.
In fact, Cleary confirms that, many times, “...organ failure is the result of energy failure on a cellular level because of oxidation damage of the mitochondrial membranes..." [91, p 164]. Furthermore, compelling evidence exists that ROS-induced mitochondrial damage may be a significant cause of physiologic dysfunction due to the subsequent loss of cellular energy [38]. Mitochondrial damage and dysfunction is common in CFS, FMS, and RA, producing a great deal of their characteristic biological markers, such as decreased ATP production and energy levels, continual free radical production and damage, and DNA fragmentation. Acaí has been shown to combat these effects via its high concentration of niacin, a B vitamin essential for ATP production in the mitochondria, and an extremely high dose of the phospholipids and phytosterols needed to repair damaged cell membranes [38, 77, $89,92,93]$.

\section{DNA Repair Mediation}

DNA damage represents perhaps one of the most imminent threats for cellular death and chronic disease in the human body. This damage often occurs in the form of fragmentation as a result of severe oxidative stress, prolonged inflammation, and compromised membrane integrity and is common to all three conditions [38, 77, 91, 93, 94]. The antiinflammatory, antioxidant, and enzyme-like activity properties of acai provide strong evidence of its ability to prevent DNA damage and encourage the body's natural DNA repair cell cycle $[71,77,78,83]$.

\section{Discussion}

Treatment for inflammatory conditions varies depending upon the condition; however, research suggests, and patients confirm, that many of the mainstream modalities provide little relief, and most fail to attack the variety of underlying factors involved. In fact, most medications are aimed at treating the obvious symptoms, such as pain, sleep disturbances, and palpable inflammation $[3,5,95,96]$ rather than the pathologies behind them. According to Dr. Robert Shmerling of Harvard Medical School, “...it's hard to predict which, if any, medication will be helpful... because many medications may cause side effects and may not work...it's important to explore other treatment options" [95].

Front-line medications for FMS, CFS, and RA include non-steroidal anti-inflammatory drugs (NSAIDs), disease-modifying anti-rheumatic drugs (DMARDs), antidepressants, corticosteroids, sleep medications, muscle relaxants, and narcotics [5]. Though these drugs may provide short-term relief from symptoms, many carry dangerous side effects and prove themselves ineffective after a relatively abbreviated period of time. For example, researchers and physicians from Harvard Medical School warn that certain anti-inflammatories boast some severe risks: "...infection, rupture of tendons around the joint, thinning of the skin near the injection site, and damage to the blood supply of the underlying bone, which can result in bone death" [97]. DMARDs, often given in the treatment of RA, often inflict such side effects as "...nausea and vomiting, rash, mild hair loss, headache, mouth sores, and muscle aches." Some patients undergoing treatment for these conditions also experience gastrointestinal bleeding, vital organ damage, decreased immune function, sepsis, lung disease, hepatitis B reactivation, encephalopathy, and muscle wasting [5].

Ongoing medical research indicates that ensuring optimal vitamin $\mathrm{D}$ levels and incorporating powerful antioxidants into mainstream treatment for FMS, CFS, and RA could prove useful [43]. The literature confirms that acaí is quite adept in its ability to remedy many of the biological abnormalities characteristic of all three conditions, and though few human trials have been conducted, current research 
suggests that the supplement carries no known side effects or drug interactions $[98,99]$.

Acaí, now considered part of the "superfood family," sits among some of the most powerful natural foods in terms of the promotion of health and wellness. Boasting an ORAC value of 1,027 , acaí is not only an effective antioxidant and inflammatory mediator, it also is more adept at promoting DNA repair, preventing cellular damage, and protecting cellular integrity than many of its superfood counterparts [76, 77, 100-102].

\section{Considerations for Future Research}

Certainly, further research should be conducted with regard to acaí , proper dosage guidelines, the most effective form of administration (i.e., flakes versus extract versus pulp), and optimal preparation techniques. An in-depth examination of patient-specific disease factors would be necessary to identify a target population for generalization, including age, disease severity, other health-related factors, and lifestyle components that may affect disease progression and/or severity. Carefully controlled observational studies or randomized, doubleblind, controlled studies would be appropriate to begin to assess acaí's true potential as a front-line option in the integrative treatment and management of acute and chronic inflammatory conditions.

\section{References}

1. Gill JM, Quisel $A(2005)$ Prevalence, presentation, and progression of the patient with fibromyalgia. Clin Fam Pract 7:181-190.

2. National Institute of Arthritis and Musculoskeletal and Skin Diseases. (2008) Questions \& answers about arthritis and rheumatic diseases. Bethesda: National Institutes of Health.

3. Gilliland RP. Fibromyalgia. 2009.

4. Murphree RH. Fibromyalgia. Nutrinews [serial online]. June 2006.

5. University of Maryland Medical Center. Fibromyalgia. 2008.

6. American Pain Society. Fibromyalgia update. 2009.

7. Mease $P$ (2005) Fibromyalgia syndrome: Review of clinical presentation, pathogenesis, outcome measures and treatment. J Rheumatol 32: 6-21.

8. Salemi S, Rethage J, Wollina U, Michel BA, Gay RE, et al. (2003) Detection of Interleukin $1 \beta$ (IL-1 $\beta$ ), IL-6, and Tumor Necrosis Factor- $\alpha$ in skin of patients with fibromyalgia. J Rheumatol 30: 146-150.

9. Bagis S, Tamer L, Sahin G, Bilgin R, Guler H,et al. (2005) Free radicals and antioxidants in primary fibromyalgia: An oxidative stress disorder? Rheumatol Int 25: 188-190.

10. Sprott H, Salemi S, Gay RE, Bradley LA, Alarcón GS, et al. (2004) Increased DNA fragmentation and ultrastructural changes in fibromyalgic muscle fibers. Ann Rheum Dis 63: 245-251.

11. Wilson RB, Gluck OS, Tesser JR, Rice JC, Meyer A, et al. (1999) Antipolymer antibody reactivity in a subset of patients with fibromyalgia correlates with severeity. J Rheumatol 26: 402-407.

12. The Chronic Syndrome Support Association (1999) New antibodies discovered in many fibromyalgia patients.

13. Cuatrecasas G, Riudavets C, Güell MA, Nadal A (2007) Growth hormone as concomitant treatment in severe fibromyalgia associated with low IGF-1 serum levels: A pilot study. BMC Musculoskelet Disord 8:119.

14. Fibromyalgia \& Fatigue Centers (2009) Molecular dysfunction in CFIDS/FM being revealed.

15. National Fibromyalgia Association.(2009) Urinary cortisol levels in FM. FM Online [serial online]

16. Maekawa K, Twe C, Lotaif A, Chiappelli F, Clark GT (2003) Function of $\beta$-adrenergic receptors on molecular cells in female patients with fibromyalgia. J Rheumatol 30: 364-368.

17. Purdy M (2005) Veterans of first Gulf War have more chronic fatigue, fibromyalgia.
18. Matsumoto T, Tsurumoto $T$ (2002) Inappropriate serum levels of IGF-1 and IGFBP-3 in patients with rheumatoid arthritis. Rheumatology 41: 352-353.

19. Armstrong DJ, Meenagh GK, Bickle I, Lee AS, Curran ES, et al. (2007) Vitamin $\mathrm{D}$ deficiency is associated with anxiety and depression in fibromyalgia. Clin Rheumatol. 26: 551-554.

20. Huisman AM, White KP, Algra A, Harth M, Vieth R, et al. (2001) Vitamin D levels in women with system lupus erythematosus and fibromyalgia. J Rheumatol 28 : 2535-2539.

21. Hayes CE, Nashold FE, Spach KM, Pedersen LB (2003) The immunological functions of the Vitamin D endocrine system. Cell Mol Biol 49: 277-300.

22. Cantora MT (2000) Is Vitamin D status an environmental factor affecting autoimmune disease prevalence? Exp Biol Med 223: 230-233.

23. Evengard B, Nilsson CG, Lindh G, Lindquist L, Eneroth P, et al. (1998) Chronic fatigue syndrome differs from fibromyalgia: No evidence for elevated substance $\mathrm{P}$ levels in cerebrospinal fluid of patients with chronic fatigue syndrome. Pain 78: $153-155$

24. Davis CD (2002) Inflammatory response.

25. Autoimmune Technologies (2009) The anti-polymer antibody assay (APA assay) and fibromyalgia syndrome.

26. Sendur OF, Turan Y, Tastaban E, Yenisey C, Serter M (2009) Serum antioxidants and nitric oxide levels in fibromyalgia: A controlled study. Rheumatol Int 29: 629-633.

27. Lund N, Bengtsson A, Thorborg P (1986) Muscle tissue oxygen pressure in primary fibromyalgia. Scand J Rheumatol 15: 165-173.

28. Ali M, Ali O (1999) Fibromyalgia: An oxidative-dysoxygenative disorder (ODD) J Integr Med 3: 17-37.

29. Joschonneck M, Grohmann G, Hein G, Sprott H (2000) Abnormal microcirculation and temperature in skin above tender points in patients with fibromyalgia. Rheumatology 39: 917-921.

30. Klimas NG, O’Brien Koneru A (2007) Chronic fatigue syndrome: Inflammation, immune function, and neuroendocrine interactions. Curr Rheumatol Rep 9 . 482-487.

31. Viral Immune Pathology Diagnostics (2009) Chronic fatigue syndrome.

32. The CFIDS Association of America. (2009) About CFIDS: Prevalence.

33. Centers for Disease Control and Prevention.(2006) Chronic fatigue syndrome: Who's at risk?

34. Walker V (2006) Is sleep the root of CFS evil? CFIDS Chronicle.

35. Skerkley JA. A book summary by Dr J A Skerkley (2007) [Review of the book Betrayal by the brain: The neurological basis of chronic fatigue syndrome, fibromyalgia syndrome, and related neural network disorders, by J. Goldstein].

36. Kennedy G, Spence VA, McLaren M, Hill A, Underwood C, Belch J (2003) Oxidative stress levels are raised in chronic fatigue syndrome and are associated with clinical symptoms. Free Radic Biol Med 39: 584-589.

37. Center for the Advancement of Health. (2002) Chronic fatigue syndrome linked to impaired stress response.

38. Nicolson GL, Ellithorpe R(2006) Lipid replacement and antioxidant nutritional therapy for restoring mitochondrial function and reducing fatigue in chronic fatigue syndrome and other fatiguing illnesses. J Chronic Fatigue Syndr 13: $57-68$.

39. U.S. National Library of Medicine. Mitochondrial DNA.( 2009).

40. Lord RS, Bralley JA(1994) Treatment of chronic fatigue syndrome with specific amino acid supplementation. J Appl Nutr 46: 74-78.

41. Addington JW (2002) Cytokine inflammation and management in fibromyalgia and chronic fatigue syndrome.

42. Emory University (2008) High levels of inflammatory markers in chronic fatigue syndrome patients signal increased risk of many other illnesses involving inflammation, such as diabetes \& cardiovascular disease.

43. Singh A, Naidu PS, Gupta S, Kulkarni SK (2002) Effect of natural and synthetic antioxidants in a mouse model of chronic fatigue syndrome. J Med Food 5: 211220.

44. National Center for Chronic Disease Prevention and Health Promotion Rheumatoid arthritis (2008). 
Citation: Relph A, Pesek T (2011) Açaí, Euterpe oleracea: Rethinking Treatment for Inflammatory Conditions. J Nutr Disorders Ther 1:103. doi:10.4172/2161-0509.1000103

45. Da Sylva TR, Connor A, Mburu Y, Keystone E, Wu GE (2005) Somatic mutations in the mitochondria of rheumatoid arthritis synoviocytes. Arthritis Res Ther 7: 844-851.

46. Metcalf $E$ (2009) The facts on inflammation.

47. World Health Organization. (2006)The global burden of rheumatoid arthritis in the year 2000 .

48. Costenbader KH, Karlson EW (2006)Epstein-Barr virus and rheumatoid arthritis: Is there a link? Arthritis Res Ther 8:204.

49. Oliver JE, Worthington J, Silman AJ (2006) Genetic epidemiology of rheumatoid arthritis. Curr Opin Epidemiol 18: 141-146.

50. Gupta A, Silman AJ (2004) Psychological stress and fibromyalgia: a review of the evidence suggesting a neuroendocrine link. Arthritis Res Ther 6: 98-106.

51. Cutolo M (2009) Circadian rhythms in arthritis - what are the implications for RA management.

52. ADAM Chronic fatigue syndrome. (2009).

53. Conger K (2001) Research shows dopamine plays crucial role in sleep regulation. Stanford Report [serial online].

54. Mukai E, Nagashima M, Hirano D, Yoshino S (2000) Comparative study of symptoms and neuroendocrine-immune network mediator levels between rheumatoid arthritis patients and healthy subjects. Clin Exp Rheumatol. 18: 585-590.

55. West SK, Oosthuizen JM (1992) Melatonin levels are decreased in rheumatoid arthritis. J Basic Clin Physiol Pharmacol. 3: 33-40.

56. Ram S, Blumberg D, Newton P, Anderson NR, Gama R (2004) Raised serum prolactin in rheumatoid arthritis: Genuine or laboratory artefact? Rheumatology 43: $1272-1274$

57. Dekkers JC, Geenen R, Godaert GLR, Bijlsma JWJ, van Doornen LJP (2004) Elevated sympathetic nervous system activity in patients with recently diagnosed rheumatoid arthritis with active disease. Clin Exp Rheumatol 22: 6370.

58. O'Shaughnessy MC, Vetsika EK, Inglis JJ, Carleson J, Haigh R, et al. (2006) The effect of substance $P$ on nitric oxide release in a rheumatoid arthritis model. Inflamm Res. 55: 236-240.

59. Bathon JM (2009) Rheumatoid arthritis pathophysiology.

60. Green PG (2005) Gastrin-releasing peptide, substance P and cytokines in rheumatoid arthritis. Arthritis Res Ther 7: 111-113.

61. Hitchon CA, El-Gabalawy HS (2004) Oxidation in rheumatoid arthritis. Arthritis Res Ther 6: 265-278.

62. Malone DG, Wahl SM, Tsokos M, Cattell H, Decker JL, Wilder RL (1984) Immune function in severe, active rheumatoid arthritis: A relationship between peripheral blood mononuclear cell proliferation to soluble antigens and synovia tissue immunohistologic characteristics. J Clin Invest 74: 1173-1185.

63. Cordain L, Toohey L, Smith MJ, Hickey MS (2000) Modulation of immune function by dietary lectins in rheumatoid arthritis. Br J Nutr 83: 207-217.

64. Malaviya AM (2006) Cytokine network and its manipulation in rheumatoid arthritis. J Assoc Physicians of India 54.

65. Kinne RW, Brauer R, Stuhlmuller B, Palombo-Kinne E, Burmester G (2000) Macrophages in rheumatoid arthritis. Arthritis Res Ther 2: 189-202.

66. Dessein PH, Stanwix AE, Joffe BI (2002) Cardiovascular risk in rheumatoid arthritis versus osteoarthritis: Acute phase response related decreased insulin sensitivity and high-density lipoprotein cholesterol as well as clustering of metabolic syndrome features in rheumatoid arthritis. Arthritis Res Ther. 4: R5.

67. U.S. National Library of Medicine. C-reactive protein (2009).

68. Cross A, Barnes T, Bucknall RC, Edwards SW, Moots RJ (2006) Neutrophil apoptosis in rheumatoid arthritis is regulated by local oxygen tensions within joints. J Leukoc Bio 80: 521-528.

69. Marklund SL, Bjelle A, Elmgvist LG (1986) Superoxide dismutase isoenzymes of the synovial fluid in rheumatoid arthritis and in reactive arthritides. Ann Rheum Dis 45: 847-851.

70. Surapneni KM, Gopan VSC (2008) Lipid peroxidation and antioxidant status in patients with rheumatoid arthritis. Indian J Biochem Biophys 23: 41-44.
71. Pacheco-Palencia LA, Talcott ST, Safe S, Mertens-Talcott S. (2008) Absorption and biological activity of phytochemical-rich extracts from Acai (Euterpe oleracea Mart.). J Agric Food Chem. 56: 3593-3600.

72. Talcott S (2010) Acai (Euterpe oleracea).

73. Lewis JA (2008) The power of knowledge: Information transfer and Aca intensification in the peri-urban interface of Belem, Brazil. Agroforestry Systems 74: 293-302.

74. Brondizio ES, Safar C, Siqueira AD (2002) The urban market of Acai fruit (Euterpe oleracea Mart.) and rural land use change: Ethnographic insights into the role of price and land tenure constraining agricultural choices in the Amazon estuary. Urban Ecosystems 6: 67-97.

75. Del Pozo-Insfran D, Percival SS, Talcott ST. (2006) Acai (Euterpe oleracea Mart.) polyphenolics in their glycoside and aglycone forms induce apoptosis of HL-60 leukemia cells. J Agric Food Chem. 54: 1222-1229.

76. Schauss AG, Wu X, Prior RL, Ou B, Huang D, Owens J, et al. (2006) Antioxidant capacity and other bioactivities of the freeze-dried Amazonian palm berry, Euterpe oleracea Mart. (Acai). J Agric Food Chem.54: 8604-8610.

77. Spada P, Nunes de Souza GG, Bortolini GV, Henriques J, Salvador M. (2008) Antioxidant, mutagenic, and antimutagenic activity of frozen fruits. J Med Food.11: 144-151

78. Lila MA (2004) Anthocyanins and human health: An in vitro investigate approach. J Biomed Biotechnol.5: 306-313.

79. Boon EM, Downs A, Marcey D. (2001) Catalase: H2O2 Oxidoreductase.

80. Worthington Biochemical Corporation. Superoxide dismutase. 2009

81. Gao F, Kinnula VL, Myllarniemi M, Oury TD (2008) Extracellular superoxide dismutase in pulmonary fibrosis. Antiox Redox Signal 10: 343-354.

82. U.S. National Library of Medicine. Reactive oxygen species. 2009.

83. Cleveland Clinic. Diet, exercise, stress, and the immune system. 2009.

84. Bourassa DJ (2009) Beta-sitosterol: Why eat your fruits and vegetables is good advice.

85. Shi Y (2008) Stem cells: NO to immune cells. UMDNJ Research.

86. Matheus ME, de Oliveira Fernandes SB, Silveira CS, Rodrigues VP, de Sousa Menezes F, et al. (2006) Inhibitory effects of Euterpe oleracea Mart. on nitric oxide production and iNOS expression. J Ethnopharmacol.107: 291-296.

87. Peeke $P(2006)$ Managing the impact of pain.

88. Goodman S (2007) Super foods: Cherries. Life Extension Magazine .

89. National Institutes of Health (2007) Vitamin B6.

90. Vgontas AN, Chrousos GP, Ilia Kritikou (2003) HPA axis and sleep.

91. Cleary JP (1988) The importance of oxidant injury as a cause of impaired mitochondrial oxidation in diabetes. J Orthomol Med 3: 164-174.

92. Richards B. (2008) Niacin: Can it help your cardio health?

93. Nicolson GL (2003) Lipid replacement as an adjunct therapy for chronic fatigue, anti-aging and restoration of mitochondrial function. J Am Nutraceutical Assoc 6: 22-28.

94. Wogan GN (2003) Reactive nitrogen and oxygen species derived from inflammatory cells.

95. Shmerling R. (2009) Best painkillers for fibromyalgia.

96. ADAM. (2008) Rheumatoid arthritis.

97. Robb-Nicolson C. (2009) Can I keep getting cortisone shots in my knee?

98. Cordova-Fraga T, deAraujo DB, Sanchez TA, Elias J Jr, Carneiro AAO, et al. (2004) Euterpe oleracea (Acai) as an alternative oral contrast agent in MRI of the gastrointestinal system: Preliminary results. Magn Reson Imaging 22: 389393.

99. Lee R, Balick MJ. (2008) Palms, people, and health. Explore 4: 59-62.

100. Chin YW, Chai HB, Keller WJ, Kinghorn AD. (2008) Lignans and other constituents of the fruits of Euterpe oleracea (Acai) with antioxidant and cytoprotective activities. J Agric Food Chem 56: 7759-7764. 
Citation: Relph A, Pesek T (2011) Açaí, Euterpe oleracea: Rethinking Treatment for Inflammatory Conditions. J Nutr Disorders Ther 1:103. doi:10.4172/2161-0509.1000103

Page 8 of 8

101.Spada PD, Dani C, Bortollini GV, Funchal C, Henriques JA, et al. (2009) Frozen fruit pulp of Euterpe oleracea Mart. (Acai) prevents hydrogen peroxideinduced damage in the cerebral cortex, cerebellum, and hippocampus of rats. J Med Food 12: 1084-1088.
102. De Souza MO, Silva M, Silva ME, Oliveira Rde, Pedrosa ML (2009) Die supplementation with acai (Euterpe oleracea Mart.) pulp improves biomarkers of oxidative stress and the serum lipid profile in rats. Nutrition 26: 804-810. 